\title{
Protein Kinase C Activators Inhibit the Visual Cascade in Limulus Ventral Photoreceptors at an Early Stage
}

\author{
Alain Dabdoub and Richard Payne \\ Department of Biology, University of Maryland, College Park, Maryland 20742
}

\begin{abstract}
The phosphoinositide cascade mediates visual transduction in invertebrate photoreceptors. Phospholipase C (PLC) catalyzes the hydrolysis of phosphatidylinositol bisphosphate, producing inositol trisphosphate ( $\operatorname{lnsP}_{3}$ ) and diacylglycerol (DAG). Protein kinase $C(P K C)$ is a major target of DAG in many cell types. We have used PKC activators to investigate the function of the kinase in the phototransduction cascade in Limulus polyphemus ventral photoreceptors. Extracellular application of (-)-indolactam V (0.03-30 $\mu \mathrm{M})$ or phorbol-12,13-dibutyrate (10 $\mu \mathrm{M})$ reversibly reduced the sensitivity of the electrical response of the photoreceptors to light by up to 1000 -fold. The inert stereoisomer $(+)$-indolactam $V$ and $4 \alpha$-phorbol had no effect. The effect of $(-)$-indolactam $V$ was antagonized by the PKC inhibitors bisindolylmaleimide I and Gö 6976. Coapplication of bisindolylmaleimide $\mathrm{V}$, used as a negative control compound for PKC inhibition, did not reduce the effectiveness of (-)-
\end{abstract}

In invertebrate microvillar photoreceptors, absorption of light by rhodopsin activates phospholipase C (PLC) via a GTP-binding protein (Bloomquist et al., 1988; Ranganathan et al., 1995). PLC catalyzes the hydrolysis of phosphatidylinositol bisphosphate producing inositol trisphosphate $\left(\mathrm{InsP}_{3}\right)$ and diacylglycerol (DAG) (Berridge, 1993). The role of the DAG branch of the cascade is not clear. In many cell types DAG is an activator of the enzyme protein kinase C (PKC) (Nishizuka, 1988), which in turn phosphorylates a variety of targets. PKCs make up a family of serine/ threonine kinases that are involved in the regulation of many cellular processes.

Light initiates three general processes in invertebrate microvillar photoreceptors: the activation of cation channels in the microvillar membrane (excitation), desensitization of the visual cascade (adaptation), and long-term changes in the structure of the microvillar membrane, such as the shedding and renewal of rhabdom at dawn. PKC has been proposed to play a role in all of these processes. Application of PKC activators to photoreceptors of the clam Lima evokes an inward current that shares some of the characteristics of the light-induced current (Gomez and Nasi, 1998). Studies of the Drosophila mutant inactivationno-afterpotential C (inaC), which lacks an eye-specific PKC (Smith et al., 1991), have indicated a role for PKC in adaptation

\footnotetext{
Received March 31, 1999; revised Sept. 10, 1999; accepted Sept. 15, 1999.

This work was supported by National Institutes of Health Grant EY-07743. We thank Dr. Ian Mather of the Department of Animal Sciences (University of Maryland, College Park, MD) for the use of the confocal microscope and Dr. Roger Hardie for his helpful criticism of this manuscript.

Correspondence should be addressed to Dr. Richard Payne, Department of Biology, University of Maryland, College Park, MD 20742. E-mail: rp12@umail.umd.edu.

Copyright (C) 1999 Society for Neuroscience $0270-6474 / 99 / 1910262-08 \$ 05.00 / 0$
}

indolactam V. These findings are consistent with (-)-indolactam $V$ activating $P K C$ and desensitizing the light response. Furthermore, our pharmacological results indicate that PKC activation does not appear to play a role in light adaptation. We localized the position of the target of PKC in the visual cascade. We chemically excited the cascade at various stages to determine the kinase's target. PKC activation by (-)-indolactam V decreased the light-induced elevation of intracellular calcium but had no effect on the photoreceptor's excitatory response to intracellular injection of $\operatorname{InsP}_{3}$. However, the PKC activator greatly reduced the excitation caused by GTP- $\gamma$-S injection. We propose that $\mathrm{PKC}$ inhibits the visual transduction cascade at the G-protein and/or PLC stage.

Key words: Limulus polyphemus; photoreceptor; PKC; phototransduction; phosphoinositide pathway; visual inhibition; desensitization; adaptation and response deactivation (Smith et al., 1991; Hardie et al., 1993). In the horseshoe crab Limulus polyphemus, PKC activators induce rhabdom shedding when injected into the compound lateral eye before dawn (Jinks et al., 1996). PKC activators have also been reported to reduce potassium channel activity and promote changes in photoreceptor morphology in the marine mollusc Hermissenda (Farley and Auerbach, 1986; Lederhendler et al., 1990; Etcheberrigaray et al., 1992).

Recent biochemical experiments have demonstrated putative PKC activity in Limulus photoreceptors. The addition of calcium and phospholipid to homogenates of Limulus ventral and lateral eyes resulted in the phosphorylation of several proteins, and this phosphorylation was inhibited by the PKC inhibitor peptide PKC 19-36 (Calman et al., 1996). The giant ventral nerve photoreceptors of Limulus have several advantages for a pharmacological study of the action of PKC. The physiological and anatomical consequences of illumination have been well defined. In Limulus ventral photoreceptors, light-induced production of $\operatorname{InsP}_{3}$ causes the release of calcium from intracellular stores (Brown and $\mathrm{Ru}$ bin, 1984; Payne et al., 1986b), leading to the opening of nonspecific cation channels in the plasma membrane and a resulting depolarization of the photoreceptor's membrane potential. Calcium release subsequently reduces the photoreceptors' sensitivity, initiating light adaptation (Brown and Lisman, 1975). Prolonged bright illumination also leads to profound, reversible changes in rhabdom structure (Herman, 1991). The stability of electrophysiological recordings from isolated ventral photoreceptors permits investigation of the reversibility of the effects of PKC activators, whereas the response to pulsed intracellular injection of intermediates, such as $\mathrm{InsP}_{3}$, allows the identification of the level of the visual cascade at which phosphorylation by PKC might act. 
A

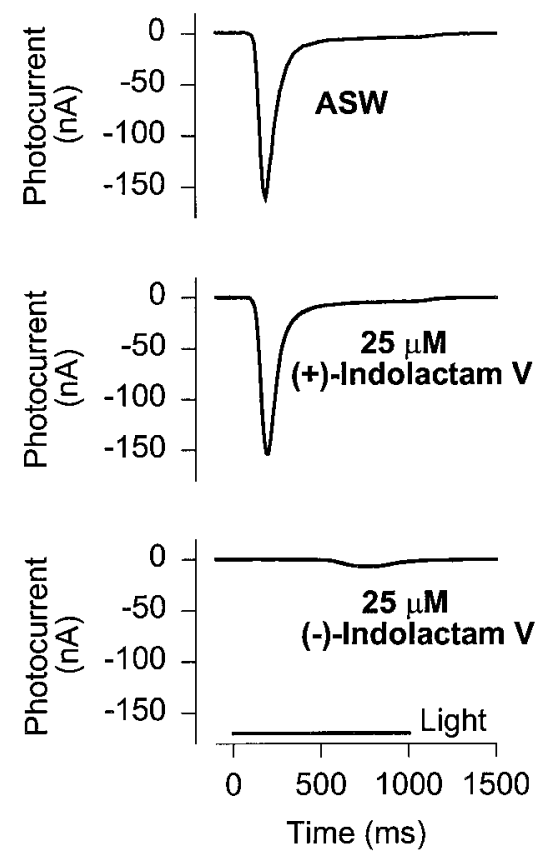

B

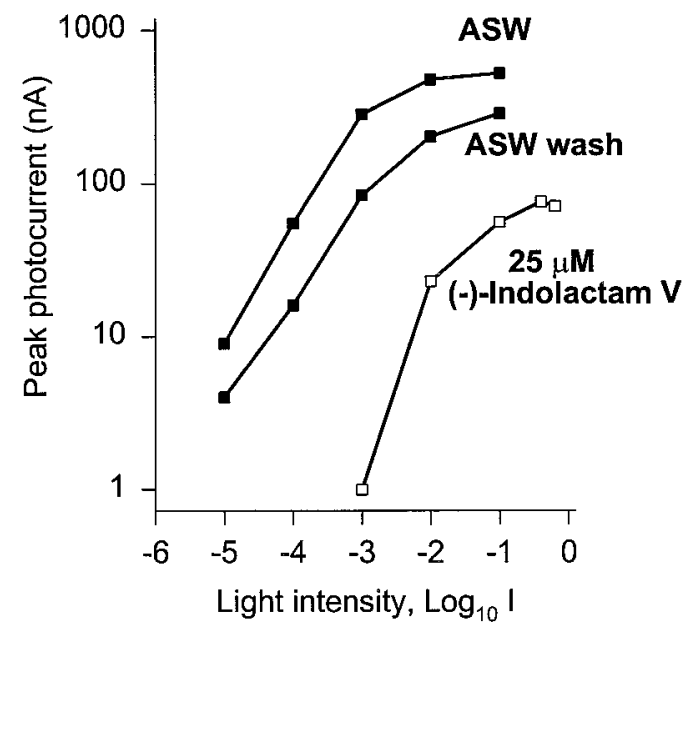

Figure 1. (-)-Indolactam V reversibly and stereospecifically inhibits the light response causing a shift in the intensity-response curve. $A$, The photoreceptor's response to a $1 \mathrm{sec}$ light stimulus $\left(\log _{10}-3\right)$ under voltageclamp conditions in ASW (top) is unaffected by $25 \mu \mathrm{M}(+)$-indolactam $\mathrm{V}$, the inactive stereoisomer (middle). The response to that same stimulus is strongly reduced $(-7 \mathrm{nA})$ in the presence of $25 \mu \mathrm{M}(-)$-indolactam V (bottom). $B,(-)$-Indolactam $\mathrm{V}$ at $25 \mu \mathrm{M}$ causes a shift in the intensity-response curve to the right by $\sim 2.5 \log _{10}$ units and a reduction in the saturated lightinduced current of $\sim 0.8 \log _{10}$ units. The effects of (-)-indolactam $\mathrm{V}$ were partially reversible after $2 \mathrm{hr}$ of washing in ASW. The light stimulus was 1 sec long, delivered at $1 \mathrm{~min}$ intervals.
Parts of this paper have been published previously (Dabdoub and Payne, 1998).

\section{MATERIALS AND METHODS}

Preparation of the ventral nerve photoreceptors was performed as described by Millecchia and Mauro (1969a,b). Nerves were placed in artificial seawater (ASW) that contained (in $\mathrm{mM}$ ): $435 \mathrm{NaCl}, 10 \mathrm{KCl}, 20$ $\mathrm{MgCl}_{2}, 25 \mathrm{MgSO}_{4}, 10 \mathrm{CaCl}_{2}$, and 10 HEPES at pH 7.3. Phorbol-12,13dibutyrate $(\mathrm{PDBu}), 4 \alpha$-phorbol, bisindolylmaleimide I and V, and Gö 6976 were obtained from Calbiochem (San Diego, CA). (-)-Indolactam $\mathrm{V}$ was obtained from both Calbiochem and Sigma (St. Louis, MO), and $(+)$-indolactam V was obtained from Sigma. All these substances were dissolved in DMSO as a $5-10 \mathrm{~mm}$ stock solution and kept at $-20^{\circ} \mathrm{C}$. Test substances were applied extracellularly by superfusion $(1 \mathrm{ml} / \mathrm{min} ; 5-10$ times bath vol) followed by a $10 \mathrm{~min}$ wait before continuing with the experiment.

White light from a $100 \mathrm{~W}$ quartz-halogen source (model 6333; Oriel Corporation, Straford, CT) illuminated the photoreceptors. The light passed through a heat filter (Schott KG3; Ealing Optics, South Natick, MA), neutral density (ND) filters, and a shutter before being focused onto the specimen plane. The intensity of the light at the specimen, with no intervening ND filters, was $80 \mathrm{~mW} / \mathrm{cm}^{2}$. Light intensities are quoted in this paper as $\log _{10}$ units of attenuation relative to this intensity. To view the preparation with an infrared-sensitive video camera, we also continuously illuminated cells by an infrared beam, created by passing a second beam of light from the quartz-halogen lamp through an infrared filter (Schott RG1000) before focusing it onto the specimen. Attenuation of the light by a $\log _{10}-8.5 \mathrm{ND}$ filter resulted in a rate of one single photon event per second.

For intracellular recording, the micropipette contained $3 \mathrm{~m} \mathrm{KCl}$. For voltage-clamp experiments, cells were impaled with a second microelectrode that also contained $3 \mathrm{M} \mathrm{KCl}$ (resistance $<10 \mathrm{M} \Omega$ ) and were voltage clamped at the dark resting membrane potential using an Axoclamp-2A amplifier (Axon Instruments, Foster City, CA). The voltage-clamped current or the membrane potential was filtered at $300 \mathrm{~Hz}$ and sampled at $1 \mathrm{kHz}$ using a Digidata 1200 (Axon Instruments) analog-to-digital board installed in a personal computer. The pCLAMP (Axon Instruments) software programs Clampex and Fetchex (version 6.0.3) were used for data acquisition.

All chemicals injected into the cell were dissolved in a carrier solution that contained $100 \mathrm{~mm}$ potassium aspartate and $10 \mathrm{~mm}$ HEPES, at $\mathrm{pH}$ 7.0. Injections were monitored using the infrared video camera (Corson and Fein, 1983a). InsP ${ }_{3}$ was obtained from Research Biochemicals (Natick, MA), dissolved into a $10 \mathrm{~mm}$ stock solution, and kept at $-20^{\circ} \mathrm{C}$.
Poorly hydrolyzable $\mathrm{L}-\mathrm{Ins}(1,3,4) \mathrm{P}_{3}$ was the gift of Dr. Barry Potter (University of Bath, Bath, United Kingdom). GTP- $\gamma$-S was obtained from Sigma, dissolved into a $1 \mathrm{~mm}$ stock solution, and kept at $-70^{\circ} \mathrm{C}$. For excitation by $\mathrm{InsP}_{3}, 10$ or $100 \mu \mathrm{M} \mathrm{InsP}_{3}$ in carrier solution was pulse pressure injected into the light-sensitive rhabdomeral lobe of the photoreceptor as described by Fein et al. (1984). For activation of G-proteins, $100 \mu \mathrm{M}$ GTP- $\gamma-\mathrm{S}$ in carrier solution was pressure injected into the cell. After multiple injections, nucleotide exchange was activated by light flashes after which the photoreceptor was dark adapted (Bolsover and Brown, 1982).

Experiments using confocal imaging and a calcium fluorescent indicator were performed as described previously (Ukhanov and Payne, 1995). Briefly, cells were injected with $500 \mu \mathrm{M}$ Oregon green-5N (Molecular Probes, Eugene, OR), a fluorescent calcium indicator dye, in carrier solution. The dissociation constant $K_{d}$ of Oregon green-5N was determined to be $18 \mu \mathrm{M}$ in a solution containing $400 \mathrm{mM} \mathrm{KCl}$ and $10 \mathrm{mM}$ HEPES at $\mathrm{pH}$ 7.0. The photoreceptors were viewed with a Zeiss LSM 410 laser-scanning confocal microscope equipped with a $488 \mathrm{~nm}$ argon laser (Uniphase) focused through a Zeiss Neofluor $10 \times, 0.3$ numerical aperture objective lens using the stationary spot recording mode of the microscope.

\section{RESULTS}

\section{Activators of PKC inhibit the electrical response to light}

(-)-Indolactam $\mathrm{V}$ is a potent and selective activator of PKC that serves as a mimic of the endogenous activator DAG. (-)Indolactam $\mathrm{V}$ activates PKC by binding to the same site on the enzyme as phorbol esters (Heikkilä and Åkerman, 1989). The specificity of (-)-indolactam $\mathrm{V}$ can be verified by performing control experiments using the stereoisomer (+)-indolactam V. Figure 1 illustrates the effects of addition of both isomers. A ventral nerve photoreceptor was dark adapted and voltage clamped to its resting membrane potential. A $1 \mathrm{sec}$ light flash (intensity, $\log _{10}-3$ ) excited an inward current, which peaked at an amplitude of $-160 \mathrm{nA}$ and then declined as adaptation proceeded (Fig. 1A, top). Superfusing the cell with the inactive stereoisomer $(+)$-indolactam $\mathrm{V}(25 \mu \mathrm{M})$ had no effect on the photoresponse (Fig. $1 \mathrm{~A}$, middle). Superfusing the cell with the same concentration of the active stereoisomer (-)-indolactam V 
A

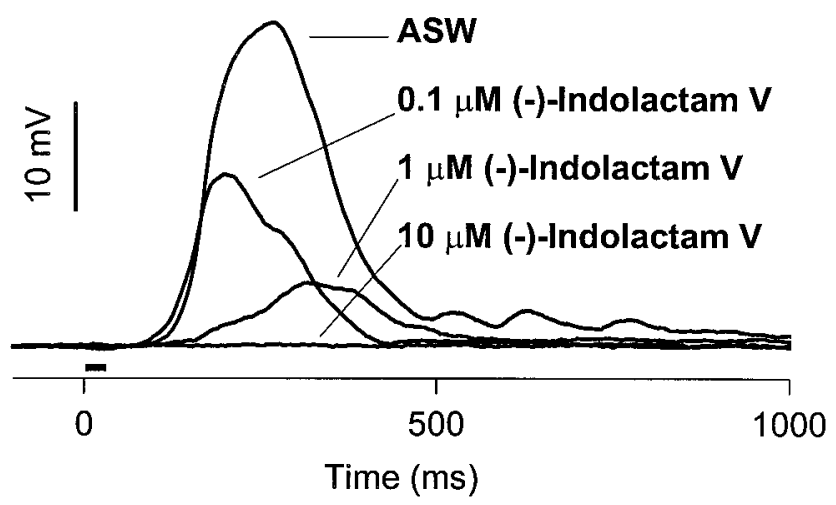

B

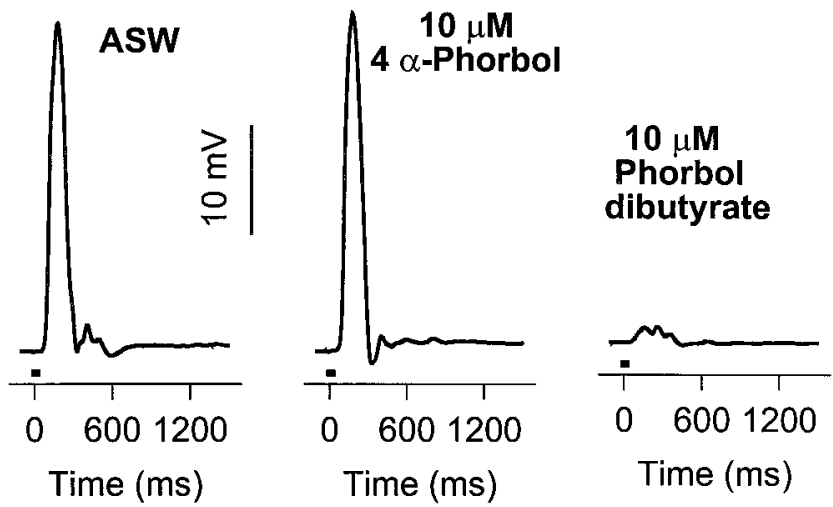

Figure 2. PKC activators decrease the photoreceptor's sensitivity to light. $A$, The receptor potential, under current-clamp recording, decreases with increasing $(-)$-indolactam $\mathrm{V}$ concentrations. $B, \mathrm{PDBu}$ also decreases the receptor potential, whereas the inactive $4 \alpha$-phorbol has no effect. The horizontal bar below the trace represents the duration of the light flash (20 msec; $\left.\log _{10}-5.4\right)$. Compounds were dissolved in DMSO and applied extracellularly to the bath. The percent DMSO was $0.18 \%$ for the different concentrations of (-)-indolactam $\mathrm{V}, 0.2 \%$ for $\mathrm{PDBu}$, and $0.1 \%$ for $4 \alpha$-phorbol.

reduced the peak response amplitude to $-7 \mathrm{nA}$ and increased its latency (Fig. $1 A$, bottom). (-)-Indolactam $\mathrm{V}$ affected all photoreceptors tested in a similar manner. The reduction of sensitivity induced by $25 \mu \mathrm{M}(-)$-indolactam $\mathrm{V}$ was analyzed in another cell by plotting the relationship between the peak response and light intensity. The effect of $25 \mu \mathrm{M}(-)$-indolactam V could be described approximately as a shift of the intensity-peak response curve to the right by $2.5 \log _{10}$ units coupled with a reduction of the saturated light-induced current by $0.8 \log _{10}$ units (Fig. $1 B, n=4$ ). The effects of $(-)$-indolactam $\mathrm{V}$ were partially reversible after a 2 hr wash.

In current-clamp recordings of membrane potential, (-)indolactam $\mathrm{V}$ decreased the photoresponse in a dose-dependent manner. Receptor potentials elicited by a $20 \mathrm{msec}$ light flash $\left(\log _{10}-5.4\right)$ decreased with increasing $(-)$-indolactam $\mathrm{V}$ concentrations (Fig. 2A). Desensitization of cells after application of $(-)$-indolactam $\mathrm{V}$ was not accompanied by significant $(>4 \mathrm{mV})$ changes in resting membrane potential or a reduction in electrical input resistance, measured with brief $-0.3-3 \mathrm{nA}$ current pulses. To check whether inhibition of the photoresponse is a common characteristic of PKC activators, we used the phorbol ester PDBu, a chemically distinct molecule that is also a specific PKC activator. Extracellular application of $10 \mu \mathrm{M}$ PDBu inhibited the photoresponse in a manner similar to that of (-)-indolactam V (Fig. $2 B, n=4)$. The effects of PDBu persisted for hours and were partially reversible after an $8 \mathrm{hr}$ wash (data not shown). The inert $4 \alpha$-phorbol had no effect on the photoresponse $(n=3)$, further confirming the specificity of the effects of PKC activators.

We examined whether the inhibition caused by DAG surrogates was indeed the result of PKC activation. We pretreated the photoreceptors with PKC inhibitors and tested the cell's response to (-)-indolactam V. We used bisindolylmaleimide I, a selective PKC inhibitor that acts as a competitive inhibitor to the ATPbinding site of PKC (Toullec et al., 1991). As a control, we used bisindolylmaleimide V (Davis et al., 1992). We also used Gö 6976, an indolocarbazole, another highly potent and selective inhibitor of PKC (Martiny-Baron et al., 1993).

For these experiments, we decided to monitor the sensitivity of the cell under current clamp, rather than two-electrode voltage clamp. Impaling the cell with a single micropipette minimized damage to the cell, resulting in less variation in the dark-adapted sensitivity to light and a greater chance that sensitivity would be stable for long time periods. However, the use of current-clamp recording complicates the analysis of the responses. For darkadapted Limulus ventral photoreceptors, the relationship between receptor potential and flash intensity is highly nonlinear. Voltage-sensitive conductances are active even during single photon signals of $>10 \mathrm{mV}$ in amplitude. Above this depolarization, rectification and the approach to the reversal potential compress the intensity-response curve (Stieve and Pflaum, 1978). This means that a small change in receptor potential amplitude might reflect a large change in the sensitivity of the cell. Rather than measure receptor potential amplitude in response to flashes of fixed intensity, we therefore quantified the effects of the inhibitors by monitoring the sensitivity of the cell as the inverse of the intensity of a $20 \mathrm{msec}$ flash required to elicit a $30 \mathrm{mV}$ criterion response. The effects of voltage-dependent conductances are then the same for all the measurements, and changes in the sensitivity of the criterion response should reflect changes in the sensitivity of the underlying photocurrent, which displays a much larger linear dynamic range. This seems to hold for our data. The 3.3 $\log _{10}$ unit decrease in sensitivity to a $30 \mathrm{mV}$ criterion response induced by $30 \mu \mathrm{M}(-)$-indolactam V (Fig. $3 B$ ) can be compared with the $2.5 \log _{10}$ unit reduction in the voltage-clamp current after $\mathrm{a}-3 \log _{10}$ unit flash induced by $25 \mu \mathrm{M}(-)$-indolactam V (Fig. $1 B$ ).

Without addition of inhibitor, sensitivity decreased progressively as (-)-indolactam $\mathrm{V}$ concentration increased from 0.03 to $30 \mu \mathrm{M}$ (Fig. $3 A$, top, $B ; n=4-6$ cells for each concentration). The inactive stereoisomer $(+)$-indolactam $\mathrm{V}$ had no effect (Fig. $3 B$, $n=3)$. The dependence of sensitivity to light on (-)-indolactam $\mathrm{V}$ concentration was then reexamined in the presence of the two PKC inhibitors (Fig. 3A,C). The PKC inhibitors alone induced a small increase in the photoreceptors' sensitivity $\left(0.5 \pm 0.4 \log _{10}\right.$ units; $n=9$, pooled data using either inhibitor). The inhibitors greatly reduced the effectiveness of (-)-indolactam V (Fig. 3A,C; $n=4-5$ for each group). In comparison with cells that were not bathed in inhibitors (Fig. $3 B$ ), the relative sensitivity was increased by $1.3 \log _{10}$ units in the presence of $1 \mu \mathrm{M}(-)$-indolactam $\mathrm{V}$ and by $2.2 \log _{10}$ units in the presence of $10 \mu \mathrm{M}(-)$-indolactam V. We obtained similar results using chelerythrine and calphostin 
A
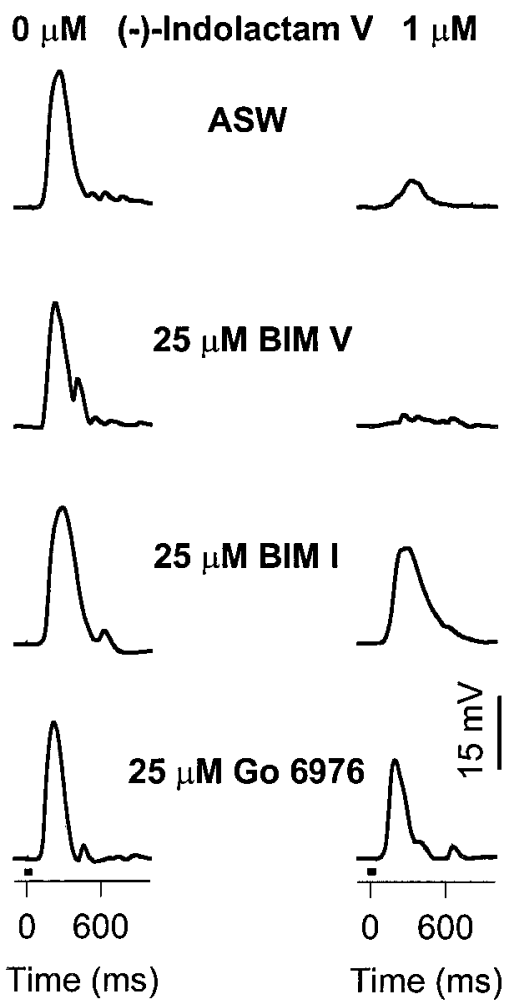

B
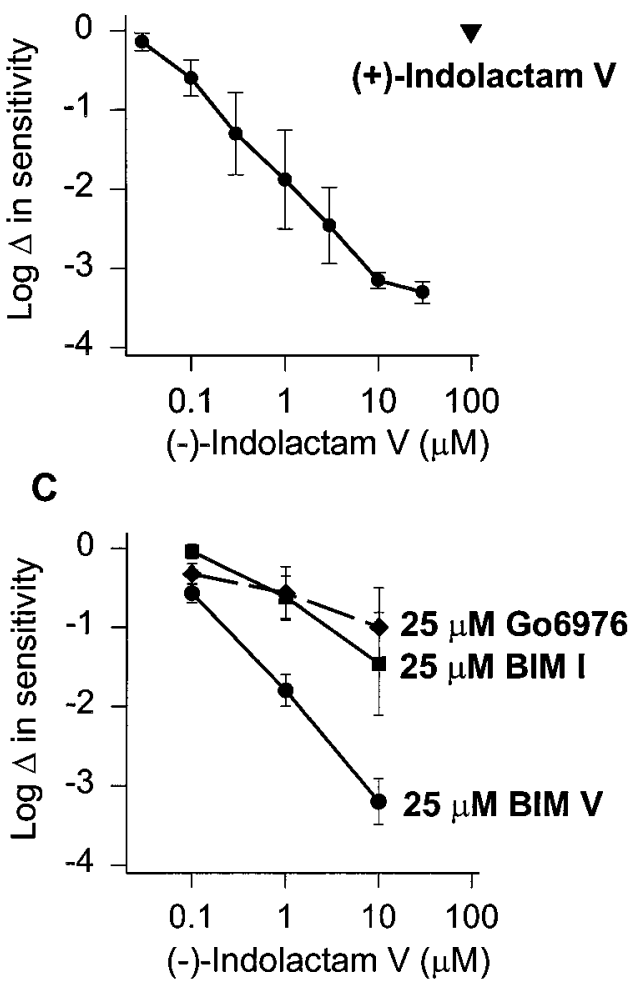

Figure 3. The effectiveness of (-)-indolactam V is decreased by selective PKC inhibitors. $A$, The PKC inhibitors bisindolylmaleimide (BIM) $I$ and Gö 6976 significantly reduced the effectiveness of $(-)$-indolactam $\mathrm{V}$ when either was coapplied. BIM $V$, a negative control compound for PKC inhibition, did not alter the effectiveness of (-)-indolactam V. The horizontal bar below the bottom receptor potential traces represents the duration of the light flash $(20 \mathrm{msec})$; the intensity of the flashes was the same before and after application of $1 \mu \mathrm{M}(-)$-indolactam V. B, Changes in sensitivity to a criterion response induced by $(-)$-indolactam $\mathrm{V}(\mathbf{)})$ were measured. A $30 \mathrm{mV}$ criterion response to a $20 \mathrm{msec}$ light flash was chosen, and the change in light intensity required to evoke the criterion response was measured $(0.5 \% \mathrm{DMSO}$ at all concentrations). At a much higher concentration, the inactive stereoisomer $(+)$-indolactam $\mathrm{V}(\boldsymbol{\nabla})$ had no effect on the photoreceptor's sensitivity to light $(1.8 \%$ DMSO). $C$, The change in the photoreceptor's sensitivity was measured for the same criterion response in the presence of specific PKC inhibitors as well as different concentrations of (-)-indolactam V. Sensitivity changes are shown relative to that measured after application of the inhibitor but before application of (-)-indolactam V and therefore do not reflect a small sensitization induced by the inhibitor alone.

C, a PKC inhibitor that interacts with the kinase's regulatory domain by competing at the binding site of DAG and phorbol esters (data not shown). Bisindolylmaleimide $\mathrm{V}$ had no significant effect on the desensitization caused by (-)-indolactam $\mathrm{V}$ (compare Fig. 3B,C). Taken together, these results are consistent with (-)-indolactam $\mathrm{V}$ activating $\mathrm{PKC}$ and desensitizing the photoresponse.

\section{PKC inhibitors do not antagonize light adaptation}

We investigated the effects of the PKC inhibitor Gö 6976 on the kinetics of the electrical response to sustained bright light and on light adaptation. Light adaptation was investigated by delivering paired $20 \mathrm{msec}$ light flashes to voltage-clamped, dark-adapted photoreceptors. The response to the second flash in the pair was greatly reduced by light adaptation (ASW, first flash, $-202 \pm 5$ $\mathrm{nA}$; second flash, $-11.3 \pm 0.6 \mathrm{nA} ; n=3$; Fig. $4 A$ ). Superfusion with ASW containing $25 \mu \mathrm{M}$ Gö 6976 resulted in a relatively small but significant ( $t$ test, $p<0.05$ ) increase in the peak response to the first flash, consistent with the increase in the sensitivity of dark-adapted photoreceptors described above. However, the PKC inhibitor had no significant effect ( $t$ test, $p>0.05$ ) on the amplitude of the light-adapted response to the second flash (25 $\mu \mathrm{M}$ Gö 6976 , first flash, $-247 \pm 19 \mathrm{nA}$; second flash, $-8.7 \pm 1.9 \mathrm{nA} ; n=$ 3; Fig. 4B). In other experiments, bisindolylmaleimide I had effects on dark-adapted sensitivity similar to those shown in Figure 4, whereas bisindolylmaleimide $\mathrm{V}$ had no significant effect (data not shown).

We were concerned that in the experiment of Figure 4, $A$ and $B$, the slightly larger amplitude of the first, dark-adapted response in the presence of the PKC inhibitor might also result in a larger adapting effect, thus masking any relief of adaptation induced by the PKC inhibitor. Therefore in experiments on three cells, we compensated for the increase in light-induced current after application of $25 \mu \mathrm{M}$ Gö 6976 by slightly reducing the intensity of the light to maintain a $-50 \mathrm{nA}$ response to a test flash. We also correspondingly reduced the intensity of a bright, adapting flash. We found that the adapting flash reduced the response to the test flashes from -50 to $-5 \pm 0.25 \mathrm{nA}$ in $\mathrm{ASW}$ and to $-7 \pm 3.5 \mathrm{nA}$ in the presence of the PKC inhibitor. Thus this modified protocol also failed to demonstrate any significant relief of light adaptation by Gö 6976 . We also used adaptation protocols in which the sensitivity of the photocurrent was examined in the presence of steady background illumination. For background intensities of $\log _{10}-3$ and $\log _{10}-2$, which desensitized voltage-clamped photoreceptors by $2-3 \log _{10}$ units, application of $25 \mu \mathrm{M}$ Gö $6976 \mathrm{had}$ no significant effect on the peak amplitude of the light-adapted responses to test flashes (data not shown). 
A

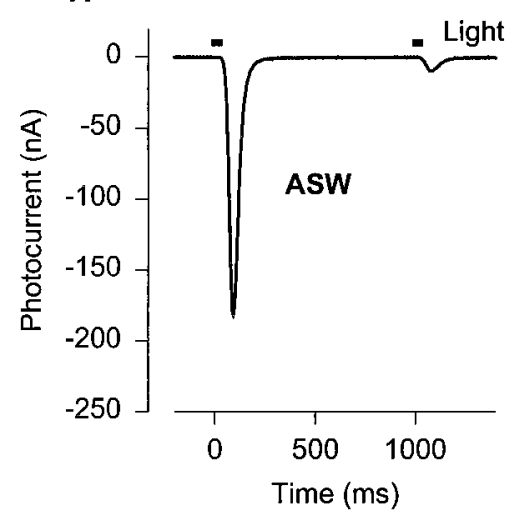

C

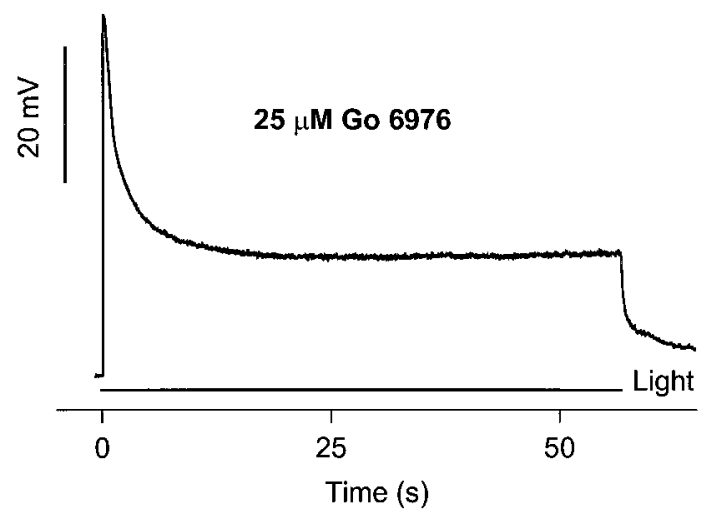

Figure 4. PKC inhibitors sensitize the photoreceptor and do not appear to affect light adaptation. $A$, Photocurrents elicited by two $20 \mathrm{msec}$ light flashes $\left(\log _{10}-3.5 ; 1 \mathrm{sec}\right.$ apart) under voltage-clamp conditions in ASW are shown. $B$, The response to the first flash is increased in the presence of $25 \mu \mathrm{M}$ Gö 6976, whereas the second flash is unchanged. $C$, Receptor potential elicited by a prolonged intense light $\left(\log _{10}-1\right)$, after overnight incubation with $25 \mu \mathrm{M}$ Gö 6976.

The rapid transition during sustained bright illumination from the initial peak transient phase of the response to the lower steady-state phase is another consequence of light adaptation. In the Drosophila inaC mutant the latter phase is missing; instead the response to sustained bright light decays slowly to baseline (Hardie et al., 1993). Ventral photoreceptors that were incubated overnight in $25 \mu \mathrm{M}$ Gö 6976 displayed receptor potentials in response to prolonged bright light $\left(\log _{10}-1\right)$ that still showed a rapid transition from a transient phase to a sustained steady-state phase (Fig. $4 C, n=4$ ). These responses are indistinguishable from control responses, as described by others (Millecchia and Mauro, 1969a).

\section{(-)-Indolactam V inhibits the phototransduction cascade at the G-protein and/or PLC stage}

Because phosphorylation of ion channels by protein kinase $\mathrm{C}$ is a well known mechanism for regulating channel activity, we examined whether the PKC acted only at a late stage in the cascade, after InsP $_{3}$-induced calcium release. In this case, the activators should not influence the amount of calcium released as a result of light stimulation. To measure light-induced calcium release, we pressure injected ventral photoreceptor cells with the calcium-

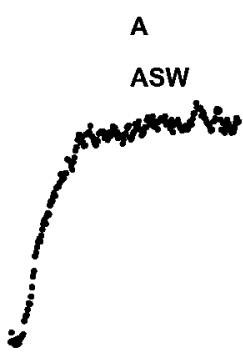

$$
\text { B }
$$

C

$25 \mu \mathrm{M}(-)$-Indolactam V

ASW wash
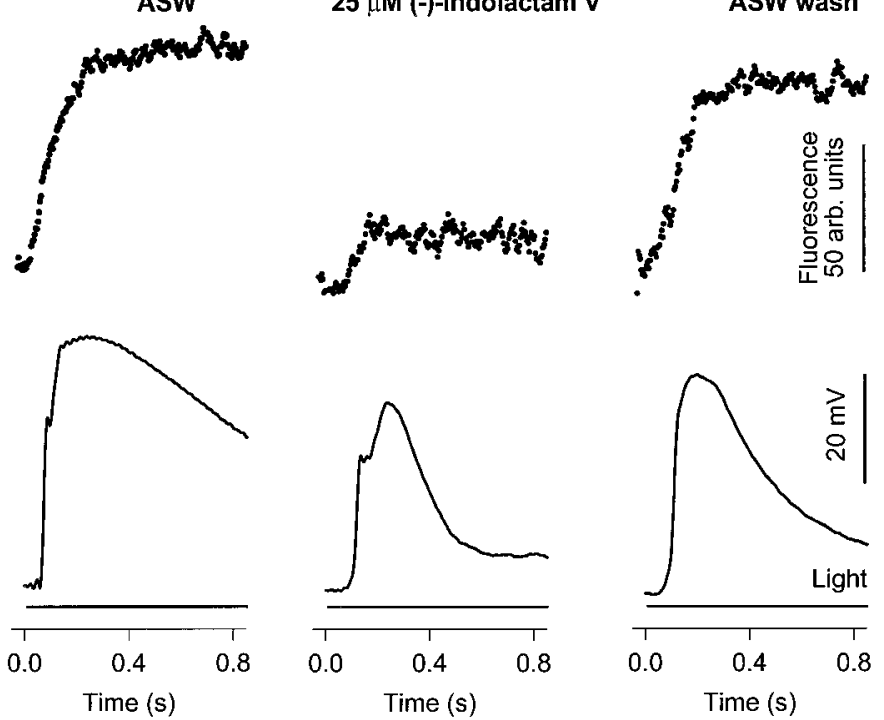

Figure 5. (-)-Indolactam V reduces light-induced calcium release. Simultaneous recordings were made of membrane potential (bottom) and the fluorescence of the calcium-indicator dye Oregon green-5N (top). The cell was pressure injected with the calcium indicator, and confocal spot measurements of Oregon green-5N fluorescence were made in the lightsensitive rhabdomeral lobe of the ventral photoreceptor. Membrane potential was sampled every $5 \mathrm{msec}$, and fluorescence was sampled every 4.5 msec. $A$, Photoreceptor bathed in ASW. $B$, After treatment with (-)indolactam V. $C$, After a $2 \mathrm{hr}$ wash with ASW. arb., Arbitrary.

sensitive fluorescent dye Oregon green-5N. Confocal spot measurements of the fluorescence of Oregon green- $5 \mathrm{~N}$ were made in the light-sensitive rhabdomeral lobe. The delayed increase in the fluorescence of calcium-sensitive dyes observed during the first few hundred milliseconds of confocal laser illumination has been shown previously to indicate light-induced release of intracellular calcium ions from intracellular stores (Ukhanov and Payne, 1995; Ukhanov et al., 1995). Figure 5 shows the simultaneous recording of the membrane potential (bottom) and Oregon green-5N signal (top). Measurements were first taken before superfusion with (-)-indolactam $\mathrm{V}$ (Fig. 5A). Both the electrical response and the calcium signal decreased in amplitude in the presence of $25 \mu \mathrm{M}$ $(-)$-indolactam $\mathrm{V}$ (Fig. $5 B, n=4)$. The effects were partially reversible after a $2 \mathrm{hr}$ wash (Fig. $5 C$ ). We conclude that (-)indolactam $\mathrm{V}$ inhibits the visual cascade at a stage that is upstream from calcium release.

We next determined whether PKC activation blocked $\mathrm{InsP}_{3^{-}}$ induced calcium release. If this was the case, then PKC activation should strongly inhibit the excitation caused by intracellular injection of $\mathrm{InsP}_{3}$, just as it inhibits the light response. Excitation by $\mathrm{InsP}_{3}$ injection was insensitive to $25 \mu \mathrm{M}(-)$-indolactam $\mathrm{V}$ (Fig. 6 , $n=5)$. In addition, $25 \mu \mathrm{M}(-)$-indolactam $\mathrm{V}$ also had no effect on repetitive bursts of depolarization caused by injecting $100 \mu \mathrm{M}$ of the poorly hydrolyzable $\operatorname{InsP}_{3}$ analog L-Ins $(1,3,4) \mathrm{P}_{3}$ (data not shown) (Riley et al., 1994). These results indicate that the primary target of PKC is upstream from $\mathrm{InsP}_{3}$-induced calcium release.

Finally, we examined the effect of PKC activation on excitation caused by the G-protein activator GTP- $\gamma$-S (Pfeuffer and Helmreich, 1975). Injecting the photoreceptor with $100 \mu \mathrm{M}$ GTP- $\gamma$-S followed by exposure to light increases the frequency of discrete 

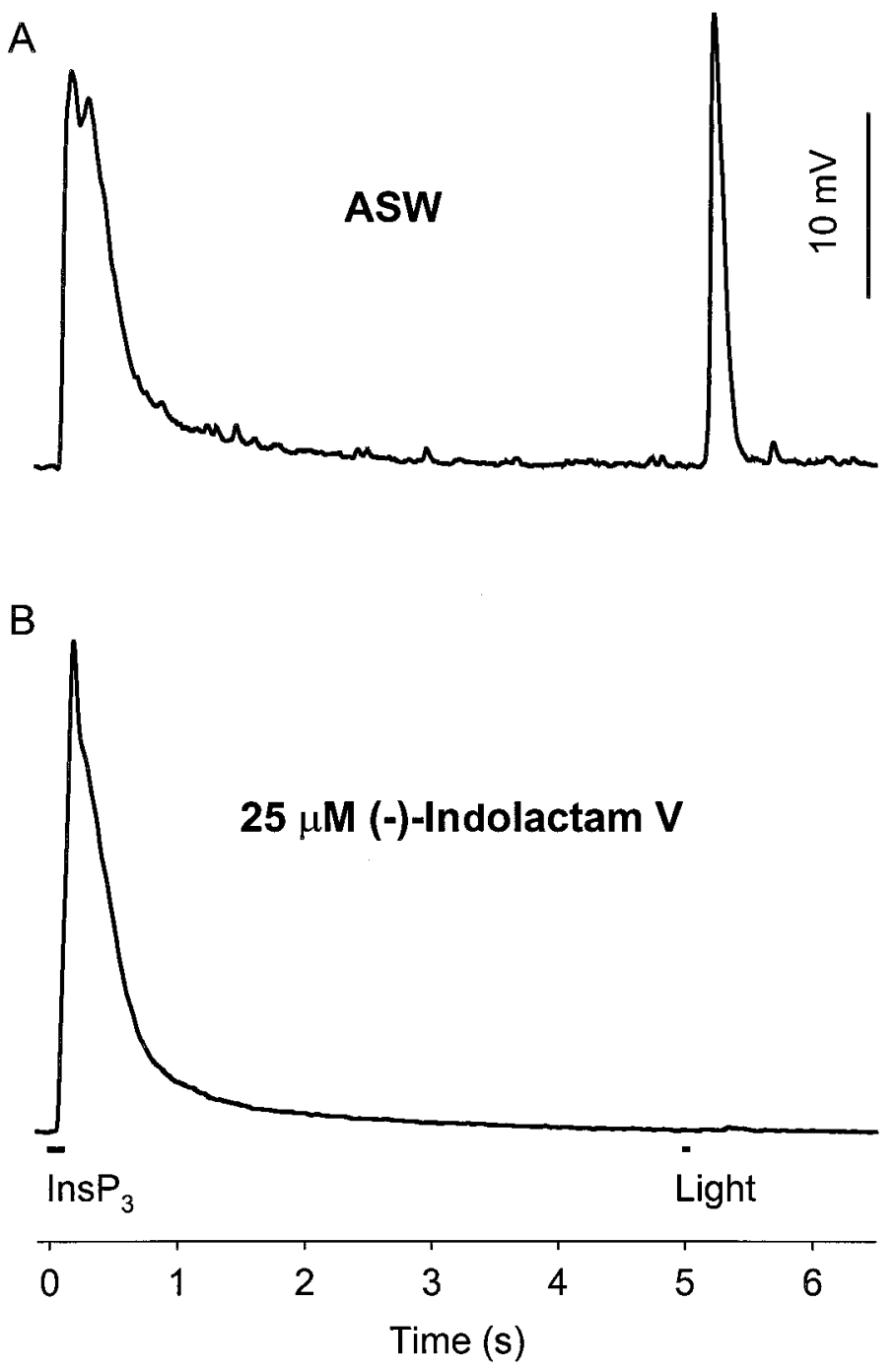

Figure 6. The desensitization of the photoreceptor by (-)-indolactam V is upstream from the $\mathrm{InsP}_{3}$ receptor in the cascade. Membrane potential was recorded during the delivery of an intracellular pressure injection $(100 \mathrm{msec})$ of $100 \mu \mathrm{M} \mathrm{InsP} \mathrm{P}_{3}$ followed by a $20 \mathrm{msec}$ light flash $\left(\log _{10}-5\right)$. $A$, Photoreceptor bathed in ASW. B, After treatment with $25 \mu \mathrm{M}(-)$ indolactam $\mathrm{V}$.

events in the dark (Fig. 7A,C) (Corson and Fein, 1983b). These events are smaller on average than spontaneous or light-induced events (Fig. 7A,B), probably because of the reduced amplification associated with the activation of the G-protein rather than rhodopsin (Kirkwood and Lisman, 1994). The induction of these events by GTP- $\gamma$-S injection is irreversible, and for large injections, the events fuse to form a noisy sustained depolarization (Corson and Fein, 1983b). The amplitude of GTP- $\gamma$-S-generated events was reduced by superfusion with the PKC activator (-)indolactam $\mathrm{V}$ (Fig. $7 D, n=4$ ), reducing the level of noise in darkness. After a $2 \mathrm{hr}$ wash with ASW, the noise recorded in darkness increased, suggesting recovery of the amplitude or frequency of GTP- $\gamma$-S-generated events, but individual events could not be distinguished (Fig. $7 E$ ). The variance of this noise was transiently reduced from 0.16 to $0.06 \mathrm{mV}^{2}$ after $20 \mathrm{msec}$ flashes of light $\left(\log _{10}-4.4\right)$ consistent with the adaptation of GTP- $\gamma-S$ induced events (Fein and Corson, 1981). We therefore conclude that desensitization by $(-)$-indolactam $\mathrm{V}$ is downstream from rhodopsin.
A AsW

darkness

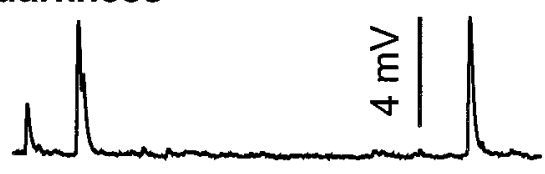

B AsW

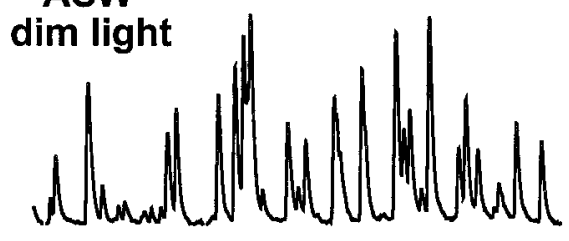

C GTP- $\gamma-S$ darkness

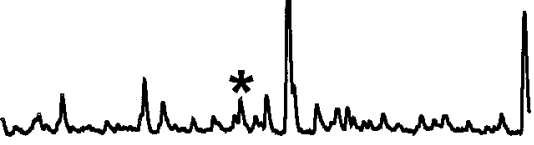

D

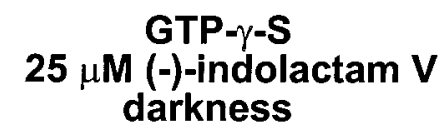

$\mathbf{E}$

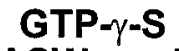

ASW wash

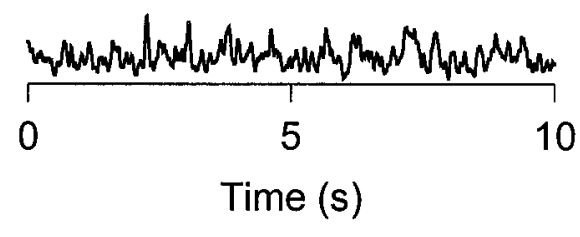

Figure 7. Desensitization by (-)-indolactam $\mathrm{V}$ is downstream from rhodopsin. $A$, Recording of membrane potential shows spontaneous depolarizing discrete events recorded in darkness. $B$, Light-induced discrete events $\left(\log _{10}-8.4\right)$ are shown. $C$, The cell was injected with GTP- $\gamma-\mathrm{S}$, and nucleotide exchange was activated by a series of light flashes, after which the cell was dark adapted. This resulted in a sustained increase in the frequency of small discrete events $(*)$. $D$, Extracellular application of $(-)$-indolactam V reduced the amplitude of the GTP- $\gamma$-S-generated events. $E$, After $2 \mathrm{hr}$ of wash, the noise level of the trace increased, consistent with the recovery of GTP- $\gamma$-S-induced events.

\section{DISCUSSION}

\section{PKC modulates photoreceptor sensitivity to light}

We have demonstrated that the light response of Limulus ventral nerve photoreceptor cells can be reversibly desensitized by several orders of magnitude by DAG surrogates as was reported in moluscan photoreceptors (Gomez and Nasi, 1998). We found this by using two distinct and potent activators of PKC, (-)indolactam $\mathrm{V}$, an indole alkaloid, and $\mathrm{PDBu}$, a phorbol ester. $\mathrm{PDBu}$ and (-)-indolactam $\mathrm{V}$ did not excite ventral photoreceptors or induce a membrane current, indicating that PKC does not mediate excitation in these photoreceptors. This is in contrast to 
Lima photoreceptors in which it was proposed recently that PKC mediates, at least in part, photoexcitation (Gomez and Nasi, 1998). The lack of an effect of the inert stereoisomer (+)indolactam $\mathrm{V}$ and $4 \alpha$-phorbol on photoreceptor sensitivity establishes the specificity of the result. In addition, PKC inhibitors antagonized the desensitization caused by (-)-indolactam V, confirming that the desensitization was indeed the result of PKC activation.

The PKC isozyme activated in this study is not an atypical PKC. There are 11 isozymes in the PKC family, all requiring phosphatidylserine (PS), a lipid found on the cytoplasmic side of membranes, for activation. These isozymes are divided into three subclasses based on their biochemical properties as follows: conventional, isozymes requiring both DAG and calcium for activation in addition to PS; novel, isozymes requiring DAG for activation in addition to PS; and atypical (aPKC), isozymes requiring only PS for activation (for review, see Newton, 1997). It is therefore possible to distinguish between the subclasses based on their cofactor requirement. The PKC activation that we described here was induced by DAG surrogates, suggesting that the PKC investigated does not belong to the aPKC subclass. Whether the kinase belongs to the conventional or novel subclass remains to be determined.

\section{PKC activators do not mimic light adaptation, nor do PKC inhibitors prevent it}

Exposure to bright light initiates a number of processes that modulate the sensitivity of a photoreceptor. The term "light adaptation" generally refers to the rapid decline in sensitivity that follows bright illumination. Light adaptation is usually accompanied by a reduced response latency and duration (Fuortes and Hodgkin, 1964). In Drosophila photoreceptors, the presence of an eye-specific PKC appears to be necessary for light adaptation (Hardie et al., 1993). Our pharmacological results do not support the concept that PKC mediates light adaptation in Limulus ventral photoreceptors. First, although (-)-indolactam V decreases the cell's sensitivity to light, it does not reduce response latency or duration (Fig. 2A; A. Dabdoub and R. Payne, unpublished observations). Second, light adaptation desensitizes the cell's response to $\operatorname{InsP}_{3}$ (Fein et al., 1984), whereas (-)-indolactam V does not (Fig. 6B). Third, although PKC inhibitors slightly sensitize the photoresponse and antagonize the effect of PKC activators, they do not reverse the decrease in sensitivity associated with light adaptation for the adaptation protocols that we describe. Finally, injection of calcium ions alone into the photoreceptor can fully mimic light adaptation (Brown and Lisman, 1975; Fein and Charlton, 1977) and suppress the response to $\mathrm{InsP}_{3}$ (Payne et al., 1986a) without any requirement for the addition of DAG or a surrogate. However, each of these results is subject to caveats, and it is not possible to conclude from these results that PKC plays no role in adaptation. In particular, desensitization mediated by PKC could be a minor component of light adaptation for the adaptation protocols that we chose. It may require more prolonged or intense illumination, or it may be redundant to calcium-mediated desensitization. It is also possible that the PKC activated by light is not as susceptible to attack by PKC inhibitors as that activated by (-)-indolactam V. Calman et al. (1996) found that the PKC inhibitors hypericin, chelerythrine, and calphostin $\mathrm{C}$ did not inhibit PKC phosphorylation of endogenous substrates in Limulus photoreceptor extracts, whereas the PKC peptide inhibitor PKC 19-36 blocked phosphorylation. In addition, we found incomplete reversal of PKC activation by the noncompet- itive inhibitors bisindolylmaleimide I and Gö 6976 (Fig. 3C), indicating that there might be a component of the putative Limulus $\mathrm{PKC}$ activity that is resistant to the inhibitors used.

\section{Site of action of PKC and its physiological role}

We have physiologically localized the site of desensitization of light-induced calcium release and depolarization by PKC activators to an intermediate that is downstream from rhodopsin but upstream from $\mathrm{InsP}_{3}$-induced calcium release. Therefore, we propose that PKC activation results in the inhibition of the phototransduction cascade at the level of the G-protein and PLC. One possibility is that PKC might be directly phosphorylating the G-protein and/or PLC in the cascade. PKC-mediated phosphorylation of PLC $\beta$ isoforms has been proposed to decrease the activation of PLC $\beta$ by the respective G-proteins (Ryu et al., 1990). Alternatively, PKC-mediated phosphorylation of the G-protein's $\alpha$ subunit may decrease its activity (Aragay and Quick, 1999).

Injection of PKC activators into the lateral eyes of Limulus induces structural changes leading to the internalization of microvillar membrane (Jinks et al., 1996), mimicking the natural shedding of microvillar membrane observed at dawn. Lightinduced internalization of the microvillar membrane has also been observed in isolated Limulus ventral nerve photoreceptors (Herman, 1991). The desensitization that we observe may therefore be a correlate or prelude to these anatomical changes. Another possibility is that PKC activation may induce the translocation from the rhabdom of one of the intermediates in the phosphoinositide cascade (Terakita et al., 1996), resulting in a slow, long-term uncoupling of the phosphoinositide cascade from rhodopsin that is distinct from the rapid calcium-mediated reduction in sensitivity that is normally associated with light adaptation.

\section{REFERENCES}

Aragay AM, Quick MW (1999) Functional regulation of $\mathrm{G}_{\alpha 16}$ by protein kinase C. J Biol Chem 274:4807-4815.

Berridge MJ (1993) Cell signaling. A tale of two messengers. Nature 365:388-389.

Bloomquist BT, Shortridge RD, Schneuwly S, Perdew M, Montell C, Steller H, Rubin G, Pak WL (1988) Isolation of a putative phospholipase C gene of Drosophila, norpA, and its role in phototransduction. Cell 54:723-733.

Bolsover SR, Brown JE (1982) Injection of guanosine and adenosine nucleotides into Limulus ventral photoreceptor cells. J Physiol (Lond) 332:325-342.

Brown JE, Lisman JE (1975) Intracellular Ca modulates sensitivity and time scale in Limulus ventral photoreceptors. Nature 258:252-254.

Brown JE, Rubin LJ (1984) A direct demonstration that inositoltrisphosphate induces an increase in intracellular calcium in Limulus photoreceptors. Biochem Biophys Res Commun 125:1137-1142.

Calman BG, Andrews AW, Rissler HM, Edwards SC, Battelle B-A (1996) Calcium/calmodulin-dependent protein kinase II and arrestin phosphorylation in Limulus eyes. J Photochem Photobiol B 35:33-44.

Corson DW, Fein A (1983a) Quantitative pressure injection of picoliter volumes into Limulus ventral photoreceptors. Biophys J 44:299-304.

Corson DW, Fein A (1983b) Chemical excitation of Limulus photoreceptors. I. Phosphate inhibitors induce discrete-wave production in the dark. J Gen Physiol 82:639-657.

Dabdoub A, Payne R (1998) Inhibition of the phototransduction cascade in Limulus ventral photoreceptors by the protein kinase $\mathrm{C}$ activator indolactam V. Soc Neurosci Abstr 24:399.

Davis PD, Hill CH, Lawton G, Nixon JS, Wilkinson SE, Hurst SA, Keech E, Turner SE (1992) Inhibitors of protein kinase C. 1. 2:3Bisarylmaleimides. J Med Chem 35:177-184. 
Etcheberrigaray R, Matzel LD, Lederhendler II, Alkon DL (1992) Classical conditioning and protein kinase $\mathrm{C}$ activation regulate the same single potassium channel in Hermissenda crassicornis photoreceptors. Proc Natl Acad Sci USA 89:7184-7188.

Farley J, Auerbach S (1986) Protein kinase C activation induces conductance changes in Hermissenda photoreceptors like those seen in associative learning. Nature 319:220-223.

Fein A, Charlton JS (1977) A quantitative comparison of the effects of intracellular calcium injection and light adaptation on the photoresponse of Limulus ventral photoreceptors. J Gen Physiol 70:591-600.

Fein A, Corson DW (1981) Excitation of Limulus photoreceptors by vanadate and by a hydrolysis resistant analogue of guanosine triphosphate. Science 212:555-557.

Fein A, Payne R, Corson DW, Berridge MJ, Irvine RF (1984) Photoreceptor excitation and adaptation by inositol 1,4,5-trisphosphate. Nature 311:157-160

Fuortes MGF, Hodgkin AL (1964) Changes in time scale and sensitivity in the ommatidia of Limulus. J Physiol (Lond) 172:239-263.

Gomez M, Nasi E (1998) Membrane current induced by protein kinase $\mathrm{C}$ activators in rhabdomeric photoreceptors: implications for visual excitation. J Neurosci 18:5253-5263.

Hardie RC, Peretz A, Suss-Toby E, Rom-Glas A, Bishop SA, Selinger Z, Minke B (1993) Protein kinase C is required for light adaptation in Drosophila photoreceptors. Nature 363:634-637.

Heikkilä J, Åkerman KEO (1989) (-)-Indolactam V activates protein kinase $\mathrm{C}$ and induces changes in muscarinic receptor functions in SH-SY5Y human neuroblastoma cells. Biochem Biophys Res Commun 162:1207-1213.

Herman KG (1991) Light-stimulated rhabdom turnover in Limulus ventral photoreceptors maintained in vitro. J Comp Neurol 303:11-21.

Jinks RN, White RH, Chamberlain SC (1996) Dawn, diacylglycerol, calcium, and protein kinase $\mathrm{C}$-the retinal wrecking crew. A signal transduction cascade for rhabdom shedding in the Limulus eye. J Photochem Photobiol B 35:45-52.

Kirkwood A, Lisman JE (1994) Determinants of single photon response variability. J Gen Physiol 103:679-690.

Lederhendler II, Etcheberrigaray R, Yamoah EN, Matzel LD, Alkon DL (1990) Outgrowths from Hermissenda photoreceptor somata are associated with activation of protein kinase C. Brain Res 534:195-200.

Martiny-Baron G, Kazanietz MG, Mischak H, Blumberg PM, Kochs G, Hug H, Marme D, Schachtele C (1993) Selective inhibition of protein kinase C isozymes by the indolocarbazole Gö 6976. J Biol Chem 268:9194-9197.

Millecchia R, Mauro A (1969a) The ventral photoreceptor cell of Limulus. II. The basic photoresponse. J Gen Physiol 54:310-330.
Millecchia R, Mauro A (1969b) The ventral photoreceptor cell of Limulus. III. A voltage-clamp study. J Gen Physiol 54:331-351.

Newton AC (1997) Regulation of protein kinase C. Curr Opin Cell Biol 9:161-167.

Nishizuka Y (1988) The molecular heterogeneity of protein kinase C and its implications for cellular regulation. Nature 334:661-665.

Payne R, Corson DW, Fein A (1986a) Pressure injection of calcium both excites and adapts Limulus ventral photoreceptors. J Gen Physiol 88:107-126.

Payne R, Corson DW, Fein A (1986b) Excitation and adaptation of Limulus ventral photoreceptors by inositol 1,4,5 triphosphate result from a rise in intracellular calcium. J Gen Physiol 88:127-142.

Pfeuffer T, Helmreich EJM (1975) Activation of pigeon erythrocyte membrane adenylate cyclase by guanylnucleotide analogues and separation of a nucleotide binding protein. J Biol Chem 250:867-876.

Ranganathan R, Malicki DM, Zuker CS (1995) Signal transduction in Drosophila photoreceptors. Annu Rev Neurosci 18:283-317.

Riley AM, Payne R, Potter BVL (1994) Unambiguous total synthesis of the enantiomers of myo-inositol 1,3,4-trisphosphate: 1L-myo-inositol 1,3,4-trisphosphate mobilizes intracellular $\mathrm{Ca}^{2+}$ in Limulus photoreceptors. J Med Chem 37:3918-3927.

Ryu SH, Kim U-H, Wahl MI, Brown AB, Carpenter G, Huang K-P, Rhee SG (1990) Feedback regulation of phospholipase C- $\beta$ by protein kinase C. J Biol Chem 265:17941-17945.

Smith DP, Ranganathan R, Hardy RW, Marx J, Tsuchida T, Zuker CS (1991) Photoreceptor deactivation and retinal degeneration mediated by a photoreceptor-specific protein kinase C. Science 254:1478-1484.

Stieve H, Pflaum M (1978) The response height versus stimulus intensity curve of the ventral nerve photoreceptor of Limulus depending on adaptation and external calcium concentration. Vision Res 18:747-749.

Terakita A, Takahama H, Tamotsu S, Suzuki T, Hariyama T, Tsukahara Y (1996) Light-modulated subcellular localization of the alphasubunit of GTP-binding protein Gq in crayfish photoreceptors. Vis Neurosci 13:539-547.

Toullec D, Pianetti P, Coste H, Bellevergue P, Grand-Perret T, Ajakane M, Baudet V, Boissin P, Boursier E, Loriolle F (1991) The bisindolylmaleimide GF $109203 \mathrm{X}$ is a potent and selective inhibitor of protein kinase C. J Biol Chem 266:15771-15781.

Ukhanov K, Payne R (1995) Light activated calcium release in Limulus ventral photoreceptors as revealed by laser confocal microscopy. Cell Calcium 18:301-313.

Ukhanov KY, Flores TM, Hsiao HS, Mohapatra P, Pitts CH, Payne R (1995) Measurement of cytosolic $\mathrm{Ca}^{2+}$ concentration in Limulus ventral photoreceptors using fluorescent dyes. J Gen Physiol 105:95-116. 